\title{
Impacts of Data Mining on Relational Database Management System Centric Business Environments
}

\author{
Md. Sajjatul Islam \\ Lecturer \\ Computer Science and Engineering (CSE) \\ East Delta University (EDU) \\ Chittagong, Bangladesh
}

\author{
Md. Zainal Abedin \\ Lecturer \\ Computer Science and Engineering (CSE) \\ University of Science and Technology Chittagong \\ (USTC) \\ Chittagong, Bangladesh
}

\begin{abstract}
The aim of this research is to discover and analyze the crucial impacts of data mining (DM) on relational database management system (RDBMS) centric business domains. The theme is to clarify the situation of having rich set of data in relational repository with the advancement of storage capacity but no strategic information and knowledge regarding relevant business areas can make the operational and decision making process more difficult and ineffective. And as a result enterprises which are associated with RDBMS can experience loss in their businesses or the specified cause can be one of the crucial factors of losing the competitive advantage in the respective fields. So this research has systematically discovered and analyzed the challenges and level of influences after the implementation of DM on that scenario which is associated with the four factors scalability, high performance, security and flexibility requirements in the respective business environments where RDBMS is acting as the predominating data and information management centre. This research has employed the secondary research and used the qualitative analysis to deduce the concepts and influence levels and significant gain with respect to those factors. As the data mining is still a young field but exploratory in nature so this research has also given the clear identification on the unresolved challenges on this track and recommendation for future works.
\end{abstract}

\section{General Terms}

Data Mining, E-business

\section{Keywords}

Data Mining (DM); Relational Database (RDB); Relational Database Management System (DBMS); Impacts; Influence; Competitive Advantage

\section{INTRODUCTION}

Data mining (DM) is the process of searching the interesting patterns or useful information to support the policy maker or knowledge worker to take the strategic decision in an organization. The main motivation behind the data mining is to turn the huge amount of data into useful and relevant information and knowledge to assist the decision maker or knowledge worker. Finding more information and knowledge from actual data can help any business organization to reap competitive advantages than its rivals. As the information industry is growing up day by day so the data mining is facing new challenges to meet the diverse requirements. So the dynamicity in business affects the database applications which in turn influence the data mining by making it more dynamic. 'Rich Data Set and Poor Information' environment in any organization can incur loss or the loss of competitive advantage. As a result ongoing researches have also been stimulated. So data mining is the rescue which is doing the job in time with high precision. Another key point is relational database management system (RDBMS) which is the most cost effective and efficient [1] data integration platform which is able to cope with the increasing demands in associated business fields and can be upgraded with the technological advances through advancement of relational model.

So this research has been carried out on the data mining impacts i.e. how the relational data mining affects business decision making process, and influences the business applications where the relational databases are used to support those business applications. so the potential issues and challenges regarding relational databases, RDBMS and business applications have been analyzed in section I. Impacts of various DM functionalities that are relevant to the relational databases have been identified and analyzed in section II. Some crucial issues such as scalability, high availability, flexibility, security, cost reduction and competitive advantages have also been analyzed in both sections and in section III. In conclusion section, the findings, challenges and recommendation have been given.

2. IMPACTS OF RELATIONAL DATABASE (RDB) AND RELATIONAL DATABASE MANAGEMENT SYSTEM (RDBMS) APPLICATIONS ON VARIOUS BUSINESS FIELDS

How the relational database and RDBMS are associated with business organizations, and the impacts on various business areas or industries have been identified and evaluated along with some relevant issues.

\subsection{Business oriented benefits with RDBMS}

RDBMS has provided tremendous business oriented advantages. Major benefits have been enumerated with business issues [2]. Firstly, warehousing of information that gives a single unified source of data as an archive. Definition of attributes helps to establish relationships between real world entities to simplify the record keeping of business operations. Also the unnecessary data redundancy reduction has saved the storage costs. Furthermore, changes to schema is possible in real world 
scenario to accommodate the changes in business applications. In addition, no language dependency allows the business organization to choose any programming language and any operating system that is convenient to them. Moreover, multiple simultaneous usages have given the opportunity to the business organizations to share their data across various departments. Besides, RDBMS has provided the organizational data security, access control, data accuracy, validity and integrity.

\subsection{Impact of RDBMS on business environments}

How RDBMS systems are influencing the various business environments have been carefully examined with the services that are available from major renowned vendor such as Oracle, Microsoft and IBM.

\subsubsection{Oracle customers' successes}

In connection with this, some of the oracle customers' successes have been investigated.

\subsubsection{Dress Barn Inc.}

Dress Barn Inc. is a national specialty apparel retailer in USA. They have achieved some significant benefits through Oracle RDBMS and its associated features [3]. These advantages are improved credit card processing system, compliance with payment card industries (PCI), risks elimination and costs reductions, higher performance, availability and scalability which helped them to the 'growth and acquisition' strategy.

\subsubsection{Telephonica O2 Germany GmbH \& Co.}

Telephonica O2 Germany GmbH \& Co. OHG, part of the Spanish telecommunications group. Telephonica S.A. serves more than 13 million mobile phone customers in Germany [4]. The core benefits are enterprise data warehouse, data extraction from more than hundred sources systems, excellent customers and business relationships, highest availability of data, long term data storage, 35 terabytes data with scalability simplified reporting, customer satisfaction, revenue projections, tariff simulation campaign management, and fraud management.

\subsubsection{Absa Group Ltd.}

Absa group Ltd. Is one of the South Africa's largest financial services group. It has gained these competitive advantages [5], such as high scalability and availability, standard business intelligence methodology, architecture tools and measurements to business strategy, ease of decision making, and elimination of the cost of delivering and printing manually generated reports.

\subsubsection{Morrison UK}

UK Morrison is one of the renowned retail supermarkets in UK. Oracle system facilitates the following area of Morrison [6]-such as operational functionality, management of the retail processes, planning processes, and supply chain management.

\subsubsection{Bharti Airtel Limited}

Bharti Airtel Limited (Airtel) is one of India's leading integrated communication service providers. they have achieved some core benefits [7], that are the enterprise wide visibility of its network assets by enabling the constant auditing of inventory data quality, user-friendly mechanism to identify and reconcile data discrepancies, improved automated solution to replace manual processes for assessing and reconciling its network assets and capacity, and improved customer satisfaction.

\subsubsection{Angelcom S. A. Bogota}

Angelcom S. A. Bogota, Columbia is a leader in collection technologies for mass passenger transit systems in Columbia. They have achieved the significant advantages [27] by deploying oracle RDBMS- which led them to get improved online access to real time transit system data across the organization. Transaction backup time has been reduced by 95 percent, and has secured the transit system's 4.5 million daily transactions.

\subsubsection{Metcash}

Metcash is a large Australian food and fast moving consumer goods marketing and distribution company. Their significant gains [8] are- improved performance sales analysis and database performance, ability to handle large volume of products, simplification of record keeping and report generation in real time, So they can take decision in a timely manner.

\subsubsection{Tesco}

Tesco is the leading retail supermarket in UK [9]. It has the enhanced global supply chain. Oracle provides [10] base for the common processes \& operations across the organization. That is the driving force to develop or expand their international business. Oracle also helped Tesco to automate, manage \& expedite the replenishment and distribution.

\subsubsection{Korean Air}

Korean Air is South Korean's national airline and its largest carrier. They have achieved some innovative benefits through oracle's RDBMS. Key benefits are [11] the ability to analyze the profitability of a flight with three hours of departure, reduced account closing and fund closing time, revenue analysis in realtime by linking the e-ticketing system with oracle, inline analysis of procurements, automated order placements and purchasing, improved facilities management and scheduling for regular site inspection, improved efficiency of production scheduling, and enhanced confidence in the accuracy of information.

\subsubsection{Microsoft customers' successes}

Microsoft is providing the Microsoft Access and Microsoft SQL server for the RDBMS. To glean some significant impacts on business, some case studies of successful customers have been analyzed.

\subsubsection{British Telecom}

BT, UK is one of the world's leading providers of communication solutions and services. It operates in 170 countries and has more than 100,000 employees. It has achieved tremendous successes [12] that are the less maintenance costs, scalability with the expansion and diverse requirements of its business, has an efficient and centralized system, greater performance and reliability that helped them to achieve business critical web application benefits, reduced workload on end user support, ability to identify the amount of development work needed in real time. As a result plan for support costs and workflow have also been easier, and billing and system support operation have been improved greatly by ensuring the high level of performance and concurrency.

\subsubsection{London Drugs}

London Drugs operate a chain of retail locations in Canada. For its PhotoLab business regarding online service for storing, sharing and ordering print of digital photographs, and supply chain business has achieved tremendous benefits for its large volume of data in PhotoLab system (about 30 Terabytes) and supply chain system[13]. These benefits are improved operations of an important and customer facing system, high level of 
reliability, safeguard for customer data from deletion or loss, reduced workloads on IT staff members and save their time with which they can involve in more productive and value added work, ability to process approximately 30,000 photo uploads, management of more than 100 million stored images, less monthly backup time and backup restore time, high level availability, and less storage cost.

\subsubsection{Reliance Petroleum}

Reliance Petroleum is the Australia's largest distribution of BP and Castrol products. Now they are reaping excellent extracts[14] such as costs reduction, improved business insight and speed up the decision making process, extended reporting capabilities to more staff that reduced the cost of extracting, sorting and accessing of data, favorable to analyze the buying pattern of customer, which can makes the business decision making process easier, and integration of the logistics information, general ledger, financial reports, credit information, stock information and more.

\subsubsection{IBM customers' successes}

IBM RDBMS is providing excellent performance, scalability, availability and reliability on various platforms (Hardware and software) in different industries[15]. Key benefits areindustry leading performance, high level of reliability, scalability and availability, coordination among various applications on various platforms such as desktop and palmtop applications can be connected to mainframe and minicomputer host databases, optimization in data management, support in various operating systems such as Windows, Linux, and Unix etc., facilitates the design, development and application deployment, greater flexibility for various platforms, strong securities to comply with Payment card industry data security standard (PCI DSS)

\subsubsection{CLS group}

CLS group that operates its business in financial market industries has deployed IBM's DB2 to fulfill these business needs[16], Which are- management of settlement risk as the global financial system needs simultaneous settlement of both sides of foreign transactions, ability to provide a system with streamlined, resilient and innovative services which can process more than 55 percent of global foreign exchange (FX) transactions, removes the FX settlement difference between payment of both side of each trade, improved efficiency of FX operation, scalable to the growing demand in market.

From the above customer successes of major RDBMS vendors shows that RDBMS is an integral part of all sorts of enterprise applications, which supports the enterprises (small to medium or medium to large in size) to regulate their core data centric applications and information management with high level of securities, scalability, availability, reliability and performance. It is an inseparable agent to reduce the overall costs and thus to maximize their profits.

\subsection{RDBMS trend in market}

According to Mullins[17] in the 2nd quarter of 2009 DBMS system gained some significant features that put impacts on the business environment. These gains are the Oracle/Sun deal added a new dimension to RDBMS performance. IBM/Exeros deal introduced a change in analyzing the disparate data sources to find out hidden information to use data centre as a competitor and strategic asset, and Oracle's acquisition of Virtual Iron Software Inc. Influenced the dynamic management of resources virtualized in data centre. Oracle's acquisition of converging, a telecommunication software provider based on Israel significantly reduced the integration and hardware costs. With this deal Information sharing among healthcare and government organizations has been improved with greater efficiency and intelligence, Sybase acquisition of Aleri Inc. , a complex event processing software provider has made Sybase DBMS more stronger in financial service sector. Major turning point came in 2nd quarter of 2010 through the announcement of SAP to acquire Sybase[18]. Main aim of that deal was to allow the business users to access the enterprise applications from unwired devices such as iPhone, blackberry etc. In 3rd quarter of 2010 IBM announced [19] its intention to acquire the Unica Corporation which is a publicly traded, Waltham, Massachusetts-based provider of marketing and web analytics solutions. This deal will reflect the automation, management and acceleration of core business processes across marketing, demand generation, sales, order processing and fulfillment.

\section{IMPACTS OF DATA MINING FUNCTIONS AND TECHNIQUES IN BUSINESS APPLICATIONS FOCUSED ON RDBMS}

\subsection{Data Mining tasks and associated methods regarding RDBMS environment}

Our concern is about the RDBMS where multi relational data mining (MRDM) is the core model[20]. Data mining tasks has been categorized into two groups [21]. One is descriptive which extracts the general description or characteristics of data in the databases. And the second one is predictive that makes some predictions based on the inference of current data in the databases. Data mining system should be able to resolve some crucial issues such as uncovering the hidden and interesting patterns, mining the all types of patterns, extracting information and knowledge at different level of abstractions, and interaction with the users so they can give hints to guide the searching of relevant patterns.

Data mining functionalities and techniques that can resolve the above issues are Class/Concept description, Mining Frequent patterns, Associations and Correlations with the user constraints [21] such as Knowledge type constraint, Data constraint, Dimension and level constraint, Interestingness constraint and Rule constraint, Classification and Prediction using IF-THEN rule, Decision Tree, Mathematical Formulae, Neural Network, Naive Bayesian Classification, Support Vector Machines and Knearest neighbor classification but for the missing or unavailable values in the data set under observation, prediction is used. In real or production environment classification and prediction are evaluated with the issues such as accuracy which is Related to confidence level, Speed regarding computation costs, and robustness. Cluster analysis: Maximum similarities among objects that will be under same cluster, and maximum dissimilarities or differences among objects in different clusters. Outlier Analysis which is able to catch the fraudulent usage of credit card. It also used for benefit theft detection. Also evolution Analysis can analyze the last several years stock market data, which in turn help them to make realistic decision on the future investment on stock market. Besides these, Data Mining system should have tight coupling to the RDB and RDBMS for greater functionalities.

\subsection{Data mining challenges in RDBMS environment}

These are the mining different kinds of knowledge in databases, interactive mining of knowledge at multiple levels of abstraction, association of background knowledge with DM technology, generalizing of data mining query language and ad- 
hoc data mining, presentation and visualization of data mining results in an easy and understandable way to the end users, handling noisy, incomplete or unavailable data in the analysis of data mining, digging down the more relevant or interesting patterns to user requirements, efficiency and scalability of data mining algorithms which are some of the core issues of data mining in the databases, devising the parallel, distributed and incremental mining algorithms, handling the security and privacy risks using integrated information systems, and mining data from global information system

\subsection{Data mining trend of applications with respect to RDBMS oriented business environment}

Data mining has already touched wide range of domains regarding various databases and other data sources. But still it is a young field as there is no common data mining system can cover almost every domain. Here we have identified some major RDBMS oriented domain specific applications [21])-

\subsubsection{Financial data analysis using data mining system}

It has already covered these services- banking services, credit services and investment services. This sector is mostly benefited from the data mining process. Under this application, the most common and critical aspects of data mining are-multidimensional data analysis and data mining from data warehouse for banking and financial data, loan payment prediction and customer credit card policy analysis, classification and clustering of customers for target marketing, and detection of money laundering and other financial crimes.

\subsubsection{Data mining for the retail Industry}

It affects the following aspects of this sector such as identification and analyzing of customers buying behavior, identification of shopping patterns and trends, improvement of quality of customer services, helps to achieve better customer retention and satisfaction, helps to trace goods consumption ratios, effective design of goods transportation and distribution policies, helps to minimize the unnecessary cost in business thus optimizing the profit.

\subsubsection{Data Mining Telecommunication sector}

The integration of telecommunication computer network, internet and many other communicating services has stimulated the rapid growth and higher competition in this area. And also it has been accumulating large amount of data from tremendous sources. So data mining has gained its significance importance to articulate some crucial facts. These benefits are clear business insight, identification of telecommunication patterns, catching fraudulent activities, optimizing the usage of resources, and improvement of quality of services

\subsubsection{Data mining for security analysis}

Business activities are greatly dependent on internet. Though it provides greater speed and flexibility in business process but has introduced and still introducing diverse security risks. So data mining has come to this field to analyze various security threats resulting from intrusions and attacks. The DM system is for the intrusion detection analysis. There are some manual systems such as misuse detection using signature technology and anomaly detection using profile strategy. But these are not dynamic and intelligent enough to detect upcoming and diverse intrusions. These also require huge amount of human efforts and time but give less accuracy. Intrusion or malicious attacks can bring significant damage to
RDBMS aspects such as confidentiality, availability of data and the integration various sites. It is still underway to maximize its scalability, efficiency and accuracy to cover diverse threat analysis

\subsection{Trend of data mining applications provided by the major data mining vendors with RDBMS provider}

Our direction is how the various business domains regarding RDBMS are influenced in progressive way with the current power of data mining system. We have chosen top three data mining vendors[22] for RDBMS data mining. These are the Oracle, IBM and Microsoft.

Oracle has brought the most challenging advances in data mining which has affected the business operations and management level as well. These are shorter information latency, Greater enhancement on performance, scalability and security of DMS, reduced the cost of developing PA/DM application, faster analysis leads the better business intelligence in real time, facilitates the business decision making process in a greater extent which can increase revenue and reduce cost, and maintaining high level of security.

Forrester Research which is an independent analyst firm has specified in their February 2010 report that oracle is a leader in predictive analysis and data mining. It has resolved the following challenges[23] in various business domains-

Retail: Customer segmentation, response modeling for the sales campaign, recommendation of next likely products, and profile of high value customers

Banking: Credit scoring, Customer profitability, and Customer targeting

Insurance: Risk factor identification, fraud detection, policy bundling, and employee retention

Healthcare: Patient procedure recommendations, patient outcome prediction, fraud detection and doctors and nurse notes analysis

Life Science: drug discovery and interaction, common factors in healthy patient, cancer cell classification, and drug safety surveillance.

Telecommunication: Customer Churn, identify cross-sell opportunities, and network intrusion detection.

Manufacturing: Root cause analysis of defects, warranty analysis, reliability analysis, yield analysis

Chemical: New compound discovery, molecule clustering, and product yield analysis.

Utilities: predict power line/equipment failure, product bundling, and consumer fraud detection

Automotive: Feature bundling for customer segments, supplier quality analysis, and problem diagnostic

Public Sector: Taxation fraud and anomalies, crime analysis, and pattern recognition in military surveillance

Microsoft has resolved the following challenges [24]-

Market Basket analysis: They are now providing the product recommendation and product placement based on the business circumstances.

Churn analysis: Gives a clear idea about the customers who are willing to switch to another service provider or willing to 
cancel the current service. This analysis provides valuable information to customer retention policy.

Market analysis: It helps enterprises to find out the group of customers who are the most loyal and profitable from them.

Forecasting: It has the business importance to identify the interrelation between sales and inventory amount. Prediction is the key to trace that rapport to remove the unnecessary loss through maximizing the performance and accuracy.

Data exploration: It is very helpful to explore existing data about customers, sales, products etc. and to analyze those data to identify the profitability and new business opportunity.

Unsupervised learning: As it is still difficult to cover all types of interesting pattern recognition using one type of data mining model. This type of feature helps to identify the previously unknown pattern to assist decision making process.

Website analysis: Helps to analyze usability of websites through the user experiences which helps them to improve, performance and user friendliness etc.

Campaign analysis: Helps to predict the target customers for particular campaign which reduces the risks of investment.

IBM is providing DB2 intelligent Miner regarding RDBMS. It has been able to resolve some significant challenges in business organizations[25]- that are Fraud detection analysis, customer segmentation, simplify market basket analysis, integration with different vendors packages, provides high performance and scalable predictive analysis, less information latency, and tight coupling with DB2.

\subsection{Privacy and security issues regarding data mining}

To do proper and exhaustive data mining it is vital to give access to shared information of companies and individuals. So there is a great to introduce the vulnerability of privacy and security of the individual and companies data. In order to mitigate these issues data mining has been employing the privacy protection and information securities. According to the Han and Kamber [21], increasing access to data or information on the web or any electronic form can intensify the threat on data mining to preserve the data privacy and security. So data mining systems are rigorously refining the focus of discovering general patterns but not disclosing or infringing the privacy of individual information. In real, some systems require access the privacy sensitive data such as banking or financial applications etc. For this some methods have been devised to remove sensitive IDs from data to protect the privacy of most individuals. But scalable privacy preserving data mining method development is still in research.

Some principles have been suggested by the Organization for Economic Co-operation and Development (OECD) to preserve the privacy of data. The principles are [21] : Purpose of specification and limitation, openness, controlling and usage, security safeguards, and individual participation. To detect inaccuracy in data is still difficult for the organization itself. To implement those principles it is obvious that the consumers should have the opportunity and flexibility to choose numerous options of how their sensitive data will be used, and should have the toll free access to those options to modify their choices. In connection with privacy and security preserving issue, data mining model has been developing and implementing the counter terrorism mining which discovers the unusual patterns, terrorist activities and fraudulent behavior. But one difficult challenge regarding these issues is the real time mining. But if the privacy preservation is the main concern in the data mining process then this research will be limited to some authorized security agents.

Some techniques have already been developed and implemented for privacy sensitive data mining. Such as database multilevel securities which ensure the access control on data at various levels, encryption such as blind signature, biometric encryption, anonymous database and intrusion detection. Recent important data mining regarding privacy is the privacy preserving data mining [26] which is also called privacy sensitive data mining or privacy enhanced data mining. Such data mining are secure multiparty computation where actual data values are encrypted but it is not suitable for the large databases, data obscuration which uses the aggregation to distort the original data values or uses the random noise. It is still difficult to develop a common framework which can be able to mitigate the privacy and security issues on sensitive data and the exploratory nature of data mining. But preventing the misuse of the data mining systems like other systems' misuse can facilitated the solution in a greater extent.

\section{FURTHER ANALYSIS OF THE LEVEL OF INFLUENCE OF DM ON RDBMS ORIENTED BUSINESS ENVIRONMENTS}

Considerable contributions have come from predominating RDBMS such as Oracle databases $10 \mathrm{~g}$ or $11 \mathrm{~g}$, Microsoft SQL server 2005 and 2008 and IBM DB2 and Informix. Some core challenging business factors such as reduction of IT cost and preserving high quality of services, performance, scalability, reliability and availability has been ensured through their outstanding contributions. Performance of relational data centric business processes and functions have been increased with DM. DMS has given the higher efficiency and dynamicity to the business processes such as operations, sales, marketing, finance, accounting, manufacturing, production, distribution, human resource management etc., and helped them to gain competitive advantages.

There is no generalized DM framework to cover the all functions regarding most of the business domains. But there are some DM products that allow to deploy other vendors packages to cover multiple functions or for flexibility for the business applications or RDBMS and OS platforms. DM for security risk analysis is one of the most challenging issues in DM area which has been identified and discussed in previous sections. Some systems such as anomaly and profiling have been employed with DMS but not sufficient for the detection of all kinds of threats in real-time. Research is going on this issue to make the system more dynamic, intelligent, efficient and accurate to cover the diverse threats. Generalization in data mining query language (DMQL) is another challenging issue. To make it industry standard, research is going on this issue. Also the exploratory nature of $\mathrm{DM}$ and business requirements poses a challenge to preserve the privacy and security of confidential data but utilize the full power of DM. Such techniques are sensitive ID hiding, concealing the private information in individual data. And DMS also maintain the privacy preserving principle of data that have been suggested by the organization for economic co-operation and development (OECD). Furthermore some privacy sensitive data mining techniques such as secure multiparty computation and data obscuration have been used. The optimization of user interaction with DMS is still a challenge. On top of that the misuse of DMS like other system and restricted access to data to some limited agents can impede the development of state of the art privacy preserving DMS. 


\section{CONCLUSION}

This research has discovered the key impacts of data mining applications in RDBMS centric business data centre or data management system. Some significant benefits and challenges have been analyzed with respect to their influences to the organizational operation levels to strategic decision making process, and thus resolving the 'Data Rich Poor Information' challenges. Furthermore the alignment of DMS with the demands regarding scalability, high performance and security issues of RDBMS oriented business environments has been identified and analyzed with respect to the major DMS vendors. More specifically it has significant gain in scalability requirements and performance improvement with intelligence in tremendous business areas. Apart from these, the preservation of security and privacy issues in analyzing RDBMS data set is not sufficient enough with the current DMS, though some techniques have already been employed but still much improvement is required. Besides these findings, some directions of research have been raised to resolve the critical challenges. These directions are the common unified DMS model with highest level of scalability to cover analyses in multiple business domains, optimization in user interactions with DMS and Data Mining Query Language (DMQL) for better understanding the business insights, and optimum solution for privacy and security sensitive DM to ensure the maximum privacy and security to cover diverse threats in real time.

\section{REFERENCES}

[1] Brodie, L., M., 2010. Data integration at scale: From relational data integration toInformation Ecosystems [Online]. Available at:<URL:http://www.michaelbrodie.com/documents/201 OAINAABSTRACT.pdf > [Accessed 9th October 2010].

[2] Satalkar ,B., 2009. Advantages of Database Management Systems [Online]. Available at: URL:http://www.buzzle.com/articles/advantages-of-

database-management-systems.html > [Accessed 20th October 2010]

[3] Oracle, 2008a. The Dress Barn, Inc. Achieves Payment Card Industry(PCI) Compliance with Oracle Advanced Security [Online].Available at: < URL: http://www.oracle.com/customers/snapshots/the-dressbarn-snapshot.pdf > [Accessed 26th October 2010]

[4] Oracle 2008b. Telefónica O2 Germany Creates 35TB Data Warehouse to Manage Data and Improve Operations [Online]. Available at: < URL: http://www.oracle.com/customers/snapshots/telefonicao2-germany-db- snapshot.pdf $>$ [Accessed 26th October 2010]

[5] Oracle, 2008c. Absa Group Delivers Business Intelligence to the Desktop to Improve Decision Making [Online]. Available at: < URL: http://www.oracle.com/customers/snapshots/absa-groupsnapshot.pdf> [Accessed 26th October 20101]

[6] IDG, 2008. Morrison's sign up oracle IT transformation [Online]. Available at:<URL:http://www.cio.co.uk/news/2610/morrisonssign-up-oracle-for-it-transform $>$ [Accessed 9th June 2010]

[7] Shores, R., 2008. Airtel Selects Oracle(r) Communications Network Integrity to OptimizeFiberOptic Network [Online]. Available at: <
URL:http://www.oracle.com/corporate/press/2008_aug/a irtel-network.html > [Accessed 26th October 2010]

[8] Oracle, 2009b. Metcash Increases Database Performance by $300 \%$, Slashes Administration Time [Online]. Available at: <URL: http://www.oracle.com/customers/snapshots/metcash-dbcase-study.pdf $>$ [Accessed 26th October 2010]

[9] IDG, 2009. CIO 100- Industries [Online]. Available at:<URL:http://www.cio.co.uk/cio100/industry $>$ [Accesse d15thJune2010]

[10] Oracle Corporate Website (2006). Oracle®Retail Warehouse Management System Underpins Tesco Global Supply Chain [Online]. Available at: <URL:http//www.oracle.com/us/corporate/press/017199 _EN>[Accessed 11th June 2010]

[11] Oracle, 2010a. Korean Air Soars to New Heights with ERP System based on Oracle EBusiness Suite R12 [Online]. Available at:<URL:http://www.oracle.com/customers/snapshots/ko rean-air-ebs-case-study.pdf > [Accessed 26th October 2010]

[12] Microsoft Corporation, 2007. Microsoft Case Studies [Online]. Available at:<URL:http://www.microsoft.com/casestudies/Case_St udy_Detail.aspx? CaseStudyID=4000001059> [Accessed 28th October 2010]

[13] Microsoft Corporation, 2010a. Retailer Improves Data Management to reduce costs and safeguard customer goodwill [Online]. Available at: < URL:http://www.microsoft.com/casestudies/MicrosoftWindows-Server- 2003/LondonDrugs/RetailerImproves-Data-Management-to-Reduce-Costs-andSafeguardCustomerGoodwill/4000008137>[Accessed 28th October 2010]

[14] Microsoft Corporation, 2010b. Petroleum Distributor Gains Better Decision Making andFaster RIO with BI Solution [Online]. Available at: < URL:http://www.microsoft.com/casestudies/MicrosoftSharepointServer/Reliance Petroleum/PetroleumDistributor-Gains-Better-Decision-Making-and-FasterROI-withBISolution/4000008023> [Accessed 28th October 2010]

[15] IBM Corporation, 2008. IBM DB2 Software [Online] Available at: $<$ URL: http://www01.ibm.com/software/data/db2/ $>$ [Accessed 27th October 2010]

[16] IBM Corporation, 2009. CLS: Revolutionising the way the world settles foreign exchange trades [Online]. Available at:<URL:http://www01.ibm.com/software/success/cssdb.nsf/CS/JSTS7VNHN C?OpenDocument\&Site=db2software\&cty=en_us > [Accessed 27th October 2010]

[17] Mullins, C., S., 2009. The Database Report-July 2009 Available <URL:http://www.tdan.com/view-featuredcolumns/10810 > [Accessed 27th October 2010]

[18] Mullins, C., S., 2010b. The Database Report- July 2010 [Online]. Available at: $<$ URL:http://www.tdan.com/viewfeatured-columns/14063 >[Accessed 27th October 2010]. 
[19] Mullins, C., S., 2010c. The Database Report-October 2010 [Online].Available

<URL:http://www.tdan.com/view-featured-

columns/14438 > [Accessed 27th October 2010]

[20] Knobbe, A., J., 2004. Multi relational Data Mining [Online].

Available

at:<

URL:http://www.kiminkii.com/thesis.pdf [Accessed 20th August 2010]

[21] Han, J., and Kamber, M., 2006. Data Mining: Concepts and Techniques. 2nd ed. New York: Morgan Kaufmann Publishers.

[22] Hwang, S., Y., 2008. DBMS Support of the Data Mining [Online].

Available

at:

URL:www.mis.nsysu.edu.tw/dbbook/DBProject2007Fall/03/report.ppt> [Accessed 20thOctober 2010]

[23] Oracle, 2010b. Oracle Data Mining $11 \mathrm{~g}$ Release 2 Available at: <URL:http://www.oracle.com/technetwork/database/opti ons/odm/overview/oow2010.pdf $>$ [Accessed 10th October 2010]
[24] ISL, 2009. Microsoft SQL Server Analysis Service Data Mining

[Online].Availableat:<URL:http://integralsolutions.web. officelive.com/MicrosoftSSAS.aspx > [Accessed 25th October 2010]

[25] IBM Corporation, 2010b.DB2 Intelligent Miner [Online].Available at:<URL:http://www01.ibm.com/software/data/iminer/ > [Accessed 15th October 2010]

[26] SlideShare Inc., 2010 .Business System Intelligence [Online]. Available at: < URL: http://www.slideshare.net/Tommy96/ppt-4035808\# $>\quad$ [Accessed 1st November 2010]

[27] Shores, R., 2009. Oracle Database $11 \mathrm{~g}$ and Options Help Organizations to ImproveApplication Performance, Availability and Scalability [Online]. Available at:

<URL:http://www.oracle.com/us/corporate/press/017829 _EN> [Accessed 26th October 2010]. 